\title{
Inorganic phosphate as a potential risk factor for chronic disease
}

\author{
Temitope Olanbiwonnu MSc, Rachel M. Holden MD
}

- Cite as: CMAJ 2018 July 3;190:E784-5. doi: 10.1503/cmaj.180525

H ealth Canada should examine the need for nutrition labelling for inorganic phosphate additives to allow more precise evaluation of the health risks associated with excess dietary phosphates.

Limiting dietary phosphate is a cornerstone in the management of patients with chronic kidney disease, a condition in which dysregulated phosphate homeostasis is associated with adverse cardiovascular outcomes. A post hoc trial analysis published in 2009 first linked phosphate concentrations to cardiovascular disease in the general population, ${ }^{1}$ and more evidence that higher serum phosphate levels, even within the normal range, are associated with adverse cardiovascular outcomes including coronary artery calcification, congestive heart failure and mortality ${ }^{2}$ - has accumulated since then. It is worth examining whether the rapidly growing and largely unregulated practice of using inorganic phosphate additives in food manufacturing may be contributing to a high burden of chronic disease, and, if so, whether it requires a national response in Canada.

A 2012 literature review estimated that the dietary intake of inorganic phosphates in Europe had doubled since 1990 largely because of the increased consumption of "ready-to-eat" processed foods. ${ }^{3}$ Inorganic phosphates are added to foods for a number of reasons: to increase water holding capacity, increase meat $\mathrm{pH}$ and slow discoloration, reduce cooking losses, retard oxidative rancidity and protect against microbial growth. Because these phosphates are in a nonorganically bound form, the excess amounts of inorganic phosphates being consumed are absorbed more readily than organic phosphates that are present naturally in food.

Although no Canadian studies exist, a study of major grocery stores in Ohio found that $44 \%$ of the best-selling grocery items contained phosphorus-containing food additives and were less expensive than foods without additives containing phosphorus. ${ }^{4}$ Excessive exposure to inorganic phosphates from consumption of processed foods may be one of many contributions to the excess burden of poverty to poor health outcomes. ${ }^{5}$ In community-based studies, including the Multi-Ethnic Study of Atherosclerosis and the Third National Health and Nutrition Examination Survey (NHANES), phosphate levels were higher in participants from lower socioeconomic backgrounds. ${ }^{6,7}$ The poorest participants in NHANES reported the lowest dietary

\section{KEY POINTS}

- Excess consumption of processed foods has been linked to increased dietary intake of inorganic phosphates.

- Higher levels of phosphate in serum have been associated with adverse cardiovascular outcomes in the general population and may contribute to chronic disease burden.

- Accurate measurement of dietary intake of inorganic phosphates is difficult because the amount of these additives is not required on food labels.

intake of phosphorus, yet had the highest serum phosphate concentrations and the highest likelihood of hyperphosphatemia. ${ }^{8}$ In addition to possible genetic variants in the regulation of phosphate, consumption of processed foods containing inorganic phosphates, which would have been unaccounted for by the dietary assessment tool, could be a partial explanation for this paradox.

The Canadian Food and Drug Regulations requires food additives to be declared in the list of ingredients of prepackaged foods. ${ }^{9}$ However, the amount is not required. If there is no specification in the regulations, then food additives must comply with the specifications in the Food Chemicals Codex ${ }^{10}$ or those of the Joint Food and Agricultural Organization of the United Nations (FAO)/World Health Organization (WHO) Expert Committee on Food Additives. ${ }^{11}$ Inorganic phosphate additives fall under the category of generally recognized as safe and as such are not subject to substantial premarket review or consistently imposed ceiling limits.

Health Canada recommends a daily dietary allowance of $700 \mathrm{mg}$ of phosphorus (or about $2100 \mathrm{mg}$ of a phosphate salt). The Third National Health and Nutrition Examination Survey showed that most sex and age groups exceeded the recommended dietary allowance. Consumption of phosphorus in Canada also likely exceeds the recommended allowance, in particular because of increasing exposure to phosphate from food additives.

However, Health Canada has not reviewed inorganic phosphate additives systematically. Previous attempts to encourage the European Food Safety Authority to restrict the amount of inorganic phosphates in food were unsuccessful because of 
insufficient high-quality data linking dietary inorganic phosphate with poor health outcomes. ${ }^{12}$ Phosphates derived from intake of inorganic phosphates are not captured by current diet assessment tools - a factor that could have a substantial influence on longitudinal studies that evaluate the association between intake of dietary phosphates and chronic disease risk.

Intake of dietary phosphates has only a weak association with serum phosphate levels, possibly owing to both the complex interplay of hormones, including parathyroid hormone (PTH), 1,25-dihydroxyvitamin D and fibroblast growth factor 23 (FGF23), that regulate phosphate homeostasis, and the lack of effective dietary tools to capture intake of inorganic phosphates. ${ }^{12}$ Typically, phosphate levels in serum do not become abnormally elevated until the estimated glomerular filtration rate (eGFR) falls below $20 \mathrm{~mL} / \mathrm{min} / 1.73 \mathrm{~m}^{2}$ (stage 4 chronic kidney disease). ${ }^{13}$ In contrast, the levels of counterregulatory hormones that act to maintain normal phosphate levels ( $\uparrow$ PTH, $F F G F 23$ and $\downarrow 1,25$-dihydroxyvitamin D) are altered much earlier in the course of renal disease, when eGFR is about $60 \mathrm{~mL} / \mathrm{min} / 1.73 \mathrm{~m}^{2}$ (onset of stage 3 chronic kidney disease). Thus, a normal level of phosphate in serum does not preclude substantial underlying dysregulation of phosphate homeostasis or substantial off-target health effects related to high levels of counterregulatory hormones. Although the implications may be greatest for people with advanced chronic kidney disease, the health risks associated with excess dietary phosphate may be underrecognized in a larger segment of the Canadian population. In a study of prevalence estimates of chronic kidney disease in Canada, 3.1\% of Canadians had stage 3 chronic kidney disease or worse; however, this number rose to $18.6 \%$ in people aged 65 years or older. ${ }^{14}$

It took almost 30 years after the recognition of the links between dietary sodium intake and chronic disease to adopt regulations that targeted food manufacturing. The first indication of recognition that Canadians should reduce their sodium intake was the 1982 iteration of Canada's Food Guide. However, nutrition labelling for dietary sodium was not introduced by Health Canada until 2003 and not mandated until 2007. Subsequently, Hypertension Canada and the Sodium Working Group lobbied for dietary sodium reduction. By 2012, Health Canada was explicitly committed to this goal and mandated that the food industry reduce sodium to targeted levels by the end of 2016. As part of this process, food additive submissions related to sodium reduction would be streamlined and expedited.

Will the perceived benefits of sodium reduction strategies simply be replaced by the harms of excessive inorganic phosphate exposure? A first step that would assist with the study of potential harms caused by excessive dietary phosphates would be mandatory inclusion of phosphate on food labels, and the disclosure of organic and inorganic phosphate amounts. Without this, Canada will not be equipped to detect any potential health risks associated with excessive exposure to phosphates.

\section{References}

1. Tonelli M, Curhan G, Pfeffer M, et al. Relation between alkaline phosphatase, serum phosphate, and all-cause or cardiovascular mortality. Circulation 2009; 120:1784-92.

2. Bai W, Li J, Liu J. Serum phosphorus, cardiovascular and all-cause mortality in the general population: a meta-analysis. Clin Chim Acta 2016;461:76-82.

3. Ritz E, Hahn K, Ketteler M, et al. Phosphate additives in food - a health risk. Dtsch Arztebl Int 2012;109:49-55.

4. León JB, Sullivan CM, Sehgal AR. The prevalence of phosphorus-containing food additives in top-selling foods in grocery stores. J Ren Nutr 2013;23:265-70.e2.

5. de Mestral C, Stringhini S. Socioeconomic status and cardiovascular disease: an update. Curr Cardiol Rep 2017;19:115.

6. Gutiérrez OM, Anderson C, Isakova T, et al.; CRIC Study Group. Low socioeconomic status associates with higher serum phosphate irrespective of race. J Am Soc Nephrol 2010;21:1953-60.

7. Gutiérrez OM, Katz R, Peralta CA, et al. Associations of socioeconomic status and processed food intake with serum phosphorus concentration in community-living adults: the Multi-Ethnic Study of Atherosclerosis (MESA). J Ren Nutr 2012;22:480-9.

8. Gutiérrez OM, Isakova T, Enfield G, et al. Impact of poverty on serum phosphate concentrations in the Third National Health and Nutrition Examination Survey. J Ren Nutr 2011;21:140-8.

9. Canadian Food and Drug Regulations. Ottawa: Minister of Justice; 2018. Available: http:/laws.justice.gc.ca/eng/regulations/C.R.C.,_c._870/page-83. html\#h-108 (accessed 2018 June 13).

10. Food Chemicals Codex. 11th ed. Rockville (MD): The United States Pharmacopeial Convention; 2018. Available: www.foodchemicalscodex.org (accessed 2018 June 13). Login required to access content.

11. Food safety. Joint FAO/WHO Expert Committee on Food Additives; 2018. Available: www.who.int/foodsafety/areas_work/chemical-risks/jecfa/en/ (accessed 2018 June 13).

12. European Food Safety Authority. Assessment of one published review on health risks associated with phosphate additives in food. EFSA J 2013;11:3444.

13. Isakova T, Wahl P, Vargas GS, et al. Fibroblast growth factor 23 is elevated before parathyroid hormone and phosphate in chronic kidney disease. Kidney Int 2011:1370-8.

14. Arora P, Vasa P, Brenner D, et al. Prevalence estimates of chronic kidney disease in Canada: results of a nationally representative survey. CMAJ 2013;185:E417-23.

Competing interests: Rachel Holden has received research grants from the Canadian Institutes of Health Research, Southeastern Ontario Medical Organization (SEAMO) Alternative Funding Plan Agreement (AFP) Innovation Fund, OPKO RENAL (investigator initiated) and Sanofi (investigator initiated). No other competing interests were declared.

This article has been peer reviewed.

Affiliation: Department of Medicine, Queen's University, Kingston, Ont.

Contributors: Both authors contributed substantially to the conception and design of the work, revised it critically for important intellectual content, gave final approval of the version to be published and agreed to be accountable for all aspects of the work.

Acknowledgement: The authors thank Corinne Babiolakis for conducting some of the literature review used in the commentary.

Correspondence to: Rachel Holden, rachel.holden@kingstonhsc.ca 